\title{
Enhancing Knowledge Acquisition in Pharmaceutical Organisations based in Pakistan
}

\author{
Muhammad Rafique \\ College of Electrical and Mechanical Engineering, National University of Science and Technology \\ Peshawar Road, Rawalpindi, Pakistan \\ E-mail: raf.sibi@ceme.nust.edu.pk \\ Richard David Evans \\ Business Information Management and Operations, University of Westminster \\ London NW1 5LS, United Kingdom \\ Rozina Khaliq \\ Islamabad, Pakistan \\ Tayyaba Hamid \\ Quetta, Pakistan
}

\begin{abstract}
Absorptive Capacity (ACAP) depicts the sequential order of activities connecting externally generated knowledge into an organisation; this involves a company's ability to acquire new knowledge from an external source, assimilate and transform it, and eventually exploit it via its industrial processes and products/services. The sandwiched role of middle managers, being interlinked between decision makers and employees, has been argued as vital to organisational success. However, their role is often viewed as having conflicts astride management i.e. between employees and decision makers. This study, using a thematic analysis approach, explores and identifies the common and conflicting role of middle managers, as viewed by different respondents in organisational hierarchies. Results, based on a sample of 33 employees operating in the Pakistan Pharmaceutical sector, indicate that conflicting roles of middle managers also persist with more common roles in organisations.
\end{abstract}

Keywords: Absorptive Capacity; Common and Conflicting Roles; Decision Makers; Knowledge Management.

\section{Introduction}

In Pakistan's Pharmaceutical industry, knowledge is typically acquired from outside the boundaries of an organisation, with the health care sector being a direct source of new knowledge and information. Similarly, the health care sector maintains a constant eye on pharmaceutical companies to understand the latest formulas for medicines, thus leading to mutual knowledge exchange between the two sectors.

Despite both sectors overlapping in knowledge acquisition requirements, the majority of the sectors organisations operate in isolation, yet expect maximum benefits from their employees; this can often lead to gaps emerging between organisations, resulting in required knowledge not always being captured and utilised to best suit company processes. Hence, it is difficult for organisations to operate in optimized environments regarding knowledge processing activities.

Improved knowledge acquisition leads to the creation of structured knowledge bases which can enhance the streamlining of corporate activities. ${ }^{1}$ In Pakistan, both sectors' traditionally focus on the internal processes to improve knowledge acquisition and less 
feedback and input is sought from external environments, though most of the knowledge is directly beneficial for both sectors. This study, therefore, aims to identify factors which attribute to knowledge acquisition processes in relation to information dependency on each other (Pharmaceutical and Health Care sector) and aims to propose a framework, shown in section 4, for improving mutual knowledge acquisition between the two sectors.

\subsection{Knowledge Acquisition}

Knowledge acquisition is the first component of the ACAP process, as highlighted by Ref. 2. The knowledge acquisition process has been debated in academia since the introduction of the Knowledge Management (KM) field, with numerous frameworks being developed for the effective acquisition of knowledge within organisations. Ref. 3 introduced the concept of 'patterns of knowledge acquisition through interaction patterns among employees'. Over time, the concept has been discussed from different perspectives, such as organisational mechanisms ${ }^{4}$, information technologies $^{5}$ and knowledge spill overs ${ }^{6}$. Some researchers believe that the process can be examined as a function of different factors, including speed, intensity and nature of an organisation. ${ }^{2}$ Ref. 2 argues that the acquisition of knowledge is fostered if an organisation focuses more on aligning their key processes with the knowledge flow internally and externally to the company.

Different types of knowledge are seen to impact on knowledge acquisition. Traditionally, studies have focused on the internal knowledge acquisition process, however over time, for companies operating in dynamic industries such as the pharmaceutical sector, the need to exploit external knowledge to remain innovative has become of core importance, while the requirement to source knowledge internally is seen as less critical. Ref. 7 explored the different roles of a shared task in the context of knowledge in business. Ref. 8 characterized the knowledge acquisition process in the context of composition of knowledge elicitation, data analysis and domain conceptualization with engineering tools in support of knowledge acquisition activities. The knowledge acquisition process has also been viewed in the context of human behaviour mechanisms. ${ }^{9,10}$

Other studies have focused on employee collaboration and joint ventures between organisations operating in the health care and pharmaceutical sector. For example, Ref. 11 highlighted the collaboration of skills and resources between health care and pharmaceutical organisations, with a focus on robust and flexible learning. Similarly, Ref. 12 explored collaborative practices between these two sectors. Although research may be identified, literature which focuses specifically on knowledge acquisition processes in Pakistan appear limited, especially those which aim to build upon knowledge acquisition, based on resources and processes of each other.

This study, therefore, aims to explore this gap in research by taking into account pharmaceutical and health care sector organisations and exploring the mutual acquisition of knowledge, based on the organisational processes and intellectual resources of each other.

\section{Methodology}

\subsection{Study Design}

This study was conducted using a qualitative and action research approach to investigate the views of employees during the knowledge acquisition process. In depth views were obtained from 33 employees using a step wise induction process. In this regard, data was collected and processed in sequential steps: Interviews; arrangement of interviews in structural forms; developing themes out of the data collected; analysis of data and evaluation of results; and development of proposed framework.

\subsection{Unit of Analysis}

The participants interviewed during this study consisted of 33 employees from large pharmaceutical companies and health sector organisations, mainly from hospitals based in Pakistan. As both sectors are traditionally involved in a continuous process to acquire new knowledge from outside their organisations in order to grow in size and refine their process, they may be seen as knowledge intensive environments.

\subsection{Study Participants}

In total, 33 employees were interviewed during our investigation. $18.1 \%$ of these held executive level positions within their organisation. $36.4 \%$ held Head of Department positions, while $45.5 \%$ were first line managers. Of the 55 participants, $63.6 \%$ percent of were 
male, while $36.4 \%$ female. In terms of educational level, $9 \%$ of interviewees held professional qualifications acquired during employment. $12.3 \%$ of interviewees held undergraduate degrees and an additional 78.7\% held a post-graduate qualification.

\subsection{Conduct of Study}

Initially, face to face interviews were conducted with ten first line managers and eight mid-level managers from both sectors, while four senior managers, specialists and doctors were consulted for initial data collection regarding the knowledge acquisition process. General information regarding the success factors relating to knowledge acquisition process was obtained, followed by 33 in-depth interviews by respondents from both sectors, which allowed for the development of the proposed framework, shown in section 4 .

The purpose of the primary research was to aid the development of the knowledge acquisition framework for two interdependent knowledge intensive environments. Therefore, in-depth interviews were conducted for further data collection purposes; this provided a strong input to the design of the framework. Feedback obtained from employees was open and generally positive. The feedback process was conducted through informal conversations with participants from both sectors. Healthy feedback was sought from both industries and proved to be equally beneficial for aiding the development of the proposed framework.

During the action planning stage, interviews were conducted in small groups, with separate face-to-face interviews being held to delve deeper into topics raised. In the implementation phase, remaining employees were interviewed. It was noted that respondents were aware of the KM processes employed within their organisations and at times it was felt that junior employees knew better than those employed in managerial positions.

During the evaluation phase, interview responses were analysed and reported on. Following the interview analysis, the proposed framework was designed and developed with allied information. The majority of interviews were conducted at the interviewees' place of work, whilst 4 were conducted at personal residences due to personal connection with the authors.

\subsection{Interview Questions}

All interviews were semi-structured and informal, following a series of 5 questions which aimed to deepen understanding of the knowledge acquisition process employed in the employee organisations. The questions asked during interview were:

- In your opinion, what are the factors contributing to knowledge acquisition within and from outside your organisation?

- How do you relate your knowledge of the Pharmaceutical Sector with the knowledge required in the Health Care Sector?

- How can interactions between both sectors be improved to acquire more structured knowledge?

- How does your organisation currently acquire Knowledge from external sources?

- Who are the key employees within your organisation assist in the acquirement of new knowledge?

\section{Results}

The results obtained from our study provide an in-depth understanding of the knowledge acquisition process and its bidirectional flow from the pharmaceutical sector to the health care sector and vice versa. The process was explored from different perspectives, keeping in view the scope of the study. Surprisingly, the majority of employees expressed similar views. The following factors and their relationships were extracted from the interview sessions and discussions with respondents.

\subsection{Knowledge acquisition - a bidirectional process}

Knowledge is required by both sectors for the effective organisation of their corporate processes; these are mutually dependent on each other. The majority of respondents showed dependency on the opposite sector in the context of knowledge acquisition. Employees were observed with strong views about knowledge acquisition and saw it as a bidirectional process, as compared to executives and senior managers. The bidirectional knowledge acquisition process was validated by executives, employees and senior managers during interview sessions.

\subsection{Internal knowledge processes}

The internal knowledge process was identified as an important variable for knowledge acquisition by both sectors. As opposed to the views of executives 
regarding bidirectional processes, the strong views were observed regarding the internal knowledge process, as compared with the views of senior managers and employees.

\subsection{Management Support}

Management support was viewed as a key factor for successful knowledge acquisition by all respondents. Employees from both sectors were strongly convinced that management support was a main attribute for successful acquisition of knowledge. Senior management held slightly different views; they considered this factor comparatively less important, as compared to the internal knowledge processes of the organisation.

\subsection{Key Information Carriers}

Key information carriers identified by all respondents from both sectors was perceived with high percentage as a main contributor to the knowledge acquisition process in organisations. However, employees and executives held equally strong views about it, as compared to senior managers.

\subsection{Key Information Handlers}

Employees whom were in possession of first-hand knowledge considered this factor a strong variable, attributing to successful knowledge acquisition inside the organisation. However, executives and senior management viewed it slightly different. Overall, all respondents identified this variable as a healthy input to the knowledge acquisition process. Fig. 1 shows the shades of these varying factors. Respondents were also asked to discuss the link between variables identified during the first round of interviews.

Knowledge acquisition was viewed by health care workers as being directly associated with the pharmaceutical sector and vice versa. Management systems were observed as a moderator to the knowledge acquisition process, though not directly involved in knowledge acquisition activities. The key information carriers of pharmaceutical sector organisations had a direct association with key information handlers of health care sector organisations and vice versa. In addition to these external factors, internal knowledge processes were observed as having a direct association with knowledge acquisition process in both types of sector.

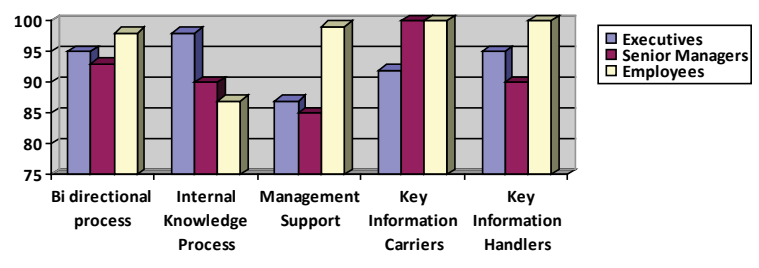

Fig. 1. Perceived Views of Respondents in Knowledge Acquisition Process.

\section{Development of the Proposed Knowledge Acquisition Framework}

Fig. 2 shows the proposed framework for bidirectional knowledge acquisition in both sectors. The flow of knowledge between the two sectors is illustrated with the required knowledge being the outcome of following the process. The knowledge acquisition element of the framework is not passive, which is similar to more traditional knowledge-intensive organisations which are dependent on the knowledge input from other organisations.

The interdependency of the two sectors organisations allow them to evaluate the newly captured knowledge through internal $\mathrm{KM}$ processes, based on its appropriateness, before acquiring it. Key information handlers and knowledge carriers in both organisations sectors play a pivotal role in the acquisition process as they become boundary spanners who act as a bridge between the two separate organisations. The role of management directly impacts on knowledge acquisition, dependent on their effective management. The closed loop of this process may be enhanced through the effective employment of organisational resources within and across both organisations. 


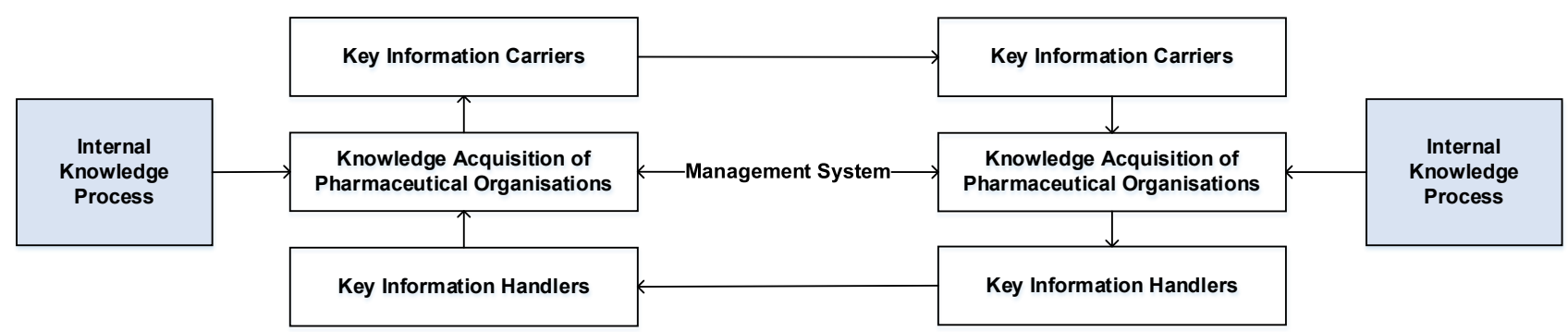

Fig. 2. Knowledge Acquisition Framework for Pharmaceutical Organisations and the Health Care Sector.

\section{Discussion}

The results of this study show that employees are fully aware of knowledge acquisition processes in both the investigated knowledge intensive sectors, although there are some minor differences in views from one employee type to another. Pharmaceutical companies require a strong input to their products, which directly comes from the health care sector and this acquisition of knowledge is not straightforward, hence it requires a systematic approach. The same may be seen in the health care sector, as they require updated knowledge about the latest trends in pharmaceutical research as they are continuously engaged in addressing growing diseases with the passage of time. Both may be seen as dependent on each other and this creates the need to establish a constant knowledge acquisition process to enable the mutual flow of knowledge between the two sectors.

Findings highlight the importance of key informers and key knowledge process handlers. Both sectors were seen as strongly linked. Information from both sectors is required by one another to operate successfully and this should be bonded together to enable greater collaboration and effective knowledge processing activities inside organisations.

The knowledge acquisition process, being dependent on internal knowledge processes, may be aligned with the day to day routine activities and also with the changing knowledge environment outside the organisation. Management systems, which enable the free flowing of information and knowledge between the two sectors was seen to have a moderating impact, as it fosters knowledge acquisition activities. The concern, however, related to the management of the knowledge acquisition process and this should not be ignored.

\section{Conclusions}

Healthy responses were received from employees, senior managers and executives in both sectors regarding knowledge acquisition processes highlighting the importance of the mutual transfer of knowledge between the two sectors. The acquisition of knowledge by one sector is dependent on the direct inputs from the other, thus highlighting the interdependent behaviour of both sectors. Employees with key information are proved to be sandwiched between the two sectors.

The result of this study highlight that management support is required during the entirety of knowledge processing activities, especially during the acquisition of knowledge from outside environments. Results indicate that knowledge acquisition activities do not work well inside the organisation.

\subsection{Limitations}

This study has focused on the development of a proposed framework, shown in section 4, for mutual knowledge acquisition by pharmaceutical and health care sector organisations which are closely related with each other during the knowledge acquisition process. The results of this study may differ if conducted in other sets of organisation type due to various reasons, including less dependency on each other. Moreover, this study was conducted in Pakistan and results in other countries may differ, especially in developed countries.

\subsection{Future Work}

The process of absorptive capacity was explored in the context of knowledge acquisition only. Other dimensions may be studied further. Finally, this study has been conducted from the perspective of two independent sectors which may be expanded on to study different departments in the same organisation. 


\section{References}

1. W.M. Cohen and D. Levinthal, 'Absorptive Capacity: A New Perspective on Learning and Innovation', Administrative Science Quarterly, Vol. 35, no. 1, 1990 pp. 128-152.

2. S.A. Zahra and G. George, 'Absorptive Capacity: A Review, Reconceptualization, and Extension', The Academy of Management Review, Vol. 27, no. 2, 2002 pp. 185-203.

3. I. Nonaka, 'A Dynamic Theory of Organizational Knowledge Creation', Organization Science, Vol. 5, no. 1, 1994 pp. 14-137.

4. J.J.P. Jansen, F.A.J. Van Den Bosch and H.W. Volberda, 'Managing Potential and Realized Absorptive Capacity: How do Organizational Antecedents Matter?', Academy of Management Journal, Vol. 48, no. 6, 2005 pp. 9991015.

5. W. Wang, Y. Feng, and L. Liu, The Effect of Organizational Levers and the Mediating Role of Individual Absorptive Capacity in Information System Innovation, in Proc. Hawaii International Conference on System Sciences, (Kauai, Hawaii, 2015), pp. 3780-3789.

6. H. Xu, D. Wan and Y. Sun, 'Technology Spillovers of Foreign Direct Investment in Coastal Regions of East China: A Perspective on Technology Absorptive Capacity', Emerging Markets Finance and Trade, Vol. 50, Sup 1, 2014 pp. 96-106.
7. F.M. Brazier, C.M. Jonker, J. Treur and N.J. Wijngaards, 'On the use of shared task models in knowledge acquistion, strategic user interaction and clarification agents' International Journal of Human-Computer Studies, Vol. 52, no. 1, 2000 pp. 77-110.

8. E. Motta, T. Rajan and M. Eisenstadt, 'Knowledge acquisition as a process of model refinement' Knowledge acquisition, Vol. 2, no. 1, 1990 pp. 21-49.

9. H.W. Volberda, N.J. Foss and M.A. Lyles, 'Absorbing the Concept of Absorptive Capacity: How to Realize Its Potential in the Organization Field' Journal of Organization Science, Vol. 21, no. 4, 2010 pp. 931-951.

10. D. Minbaeva, T. Pedersen, I. Björkman, C.F. Fey and H.J. Park, 'MNC knowledge transfer, subsidiary absorptive capacity, and HRM', Journal of International Business Studies, Vol. 34, no. 6, 2003 pp. 586-599.

11. W.W. Powell, 'Learning from collaboration: Knowledge and networks in the biotechnology and pharmaceutical industries', California management review, Vol. 40, no. 3, 1998 pp. 228-240.

12. F. Martin-Sanchez, I. Iakovidis, S. Nørager, V. Maojo, P. de Groen, J. Van der Lei and R. Baud, 'Synergy between medical informatics and bioinformatics: facilitating genomic medicine for future health care' Journal of biomedical informatics, Vol. 37, no. 1, 2004 pp. 30-42. 